\title{
Effects of crack-dilatancy on Rayleigh waves in fluid-saturated porous media
}

\author{
J. S. Nandal ${ }^{1}$ and T. N. Saini ${ }^{2 *}$ \\ ${ }^{1}$ Department of Mathematics, M.D. University, Rohtak, INDIA \\ ${ }^{2}$ Department of Mathematics, Government College Kalka, INDIA \\ ${ }^{2 *}$ Corresponding author: e-mail²: tns.kalka@gmail.com,e-mail ${ }^{1}$ j_s_nandal@yahoo.com
}

\begin{abstract}
The study considers the propagation of surface waves on the stress-free surface of a porous solid saturated with non-viscous fluid. The surface pores have the option of being either sealed or fully-opened. With the presence of dilatant cracks, the interior of the porous solid is characterised through three different crack-regimes, based on the connections between embedded cracks. Secular equations are derived in closed form for the propagation of Rayleigh waves in the porous media with sealed or fullyopened surface pores. The velocity of non-dispersive surface waves varies significantly with the density of cracks present. However, aspect (thickness to radius) ratio of (circular) cracks may not have much effect on the velocity of Rayleigh waves. The opening of surface pores may be an important reason for a faster propagation of Rayleigh waves in any realistic elastic medium. Finally, the dilatancy due to the growth of cracks up to their interconnection or drainage may be able to affect the velocity of Rayleigh waves quite significantly.
\end{abstract}

Keywords: Rayleigh waves, phase velocity, dilatancy, porous solid, cracks.

\section{Introduction}

The concept of porosity and dilatancy in elastic media has gained much attention in recent years. The applications of these studies cover a variety of fields including geophysics, soil mechanics, earthquake preparation and civil engineering. In particular, in the exploration of oil and gas reservoirs, it is important to predict / estimate the rock porosity, the presence of fluid in pores and the channels of fluid-flow due to abnormal pore-pressures. These micro-structural properties and in-situ rock conditions can be obtained mainly from seismic properties such as travel times (or phase / wave velocities), amplitude information (reflection / refraction coefficients) and wave polarization (motion of constituent particles). These measurable quantities are affected by the presence and saturation of pores and cracks with fluids. However, there must be some definite relationship between the seismic properties and rock characteristics such as porosity, permeability and tortuosity of porous solid matrix as well as shape, stiffness and density of cracks present. The use of modeling and inversion procedures for the interpretation of the seismic response of reservoir rocks requires an understanding of such relations.

The study of mechanical behaviour of porous media is of special importance in the seismic exploration, for the closer description of physical phenomena around the oil reservoirs. Sedimentary rocks contain water-filled pore spaces after deposition and can be modeled as water saturated porous solid. Moreover, every hydrocarbon field is also a reservoir of cracks filled with water, oil or gas. The full-dynamic theory for wave propagation in fluid-saturated porous media was developed by Biot (1956). Biot used Lagrange's equations to derive a set of coupled differential equations that govern the motions of solid and fluid phases. Biot (1962a) extended the acoustic propagation theory in the wider context of the mechanics of porous media. Biot (1962b) developed the new features of the extended theory, in more detail. This theory is obtained through a new and simplified derivation of the fundamental equations of poroelastic propagation. This, also, provides an exact procedure for the evaluation of the dynamic properties of the fluid motion relative to the solid. Since then, most of the studies on propagation in porous media are based on 
Biot's theories. Using Biot's theory, a modified Christoffel equation for the propagation of plane harmonic waves in an anisotropic fluid saturated porous media is derived by Sharma (Sharma, 2004a,b) have derived expression for the dispersion of surface waves in a general anisotropic poroelastic medium. Vashisth and Khurana (2005) studied the problem of elastic wave propagation along a cylindrical borehole embedded in an anisotropic fluid-saturated porous solid of infinite extent and derived the equations for the frequency of the surface waves corresponding to the empty and liquid-filled bore holes. Plane-strain deformation of a multilayered poroelastic half space by surface load is modeled by Singh and Rani (2006). In another study, Singh et al. (2007) presented an analytical solution of the fully coupled diffusion-deformation system of equations governing the quasi-static plane-strain deformation of a parabolic poroelastic half-space with anisotropic permeability and compressible constituents.

The effects of cracks on seismic waves are important since seismic experiments are one of the few geophysical techniques capable of examining the properties of in-situ rocks in the crust. Modifications of these cracks are the most direct effects of accumulation of stress before an earthquake. These may be the changes in orientation, density and thickness of the cracks. These modifications in the configuration of cracks in a focal region are believed to be the driving mechanism for the precursors of an earthquake. The theory of effects of cracks on the elastic solids started with the classic paper by Eshelby (1957). The wave velocities for the elastic solids containing cracks have been approximated by Garvin and Knopoff (1973, 1975a,b). O'Connell and Budiansky (1974) and Budiansky and O'Connell (1976) calculated the effects of introduction of cracks on the elastic properties of an isotropic solid using self-consistent procedure. Hudson $(1980,1981)$ developed these ideas further for dilute concentration of cracks, treating cracked solid as anisotropic one. Crampin $(1978,1984,1985,1987)$ studied various aspects of wave propagation in cracked solids with presence of aligned crack leading to anisotropy. Crampin (1987) explained the effects of stress accumulation, before an earthquake, on the modifications of the cracks present. It may be useful to estimate the possible changes in crack scenario, prior to an earthquake, as a precursor.

The effect of co-existence of cracks and pores on the overall properties of a cracked porous solid sounds a comprehensive and very significant aspect of wave motion. Sharma (1996) considered this co-existence and studied the surface waves in fluid layer overlying a cracked porous solid. Hudson et al. (1996) considered the existence of connections between otherwise isolated cracks and of small-scale porosity within the 'solid' material. They provided effective medium models for the calculation of elastic wave propagation with wavelengths greater than the dimensions of the cracks. Sharma (1999) studied the effect of crack connections on the reflection and refraction at an interface with elastic solid. Sharma and Saini (2001) studied effect of presence and modifications of cracks on the velocity anomalies in a cracked poroelastic medium. Recent observations (Crampin, 2006) of stress-aligned shearwave splitting (seismic-birefringence) show that the splitting is controlled by the stress-aligned fluid-saturated inter-granular microcracks and preferentially-orientated pores. Such pores are pervasive in most in-situ igneous, metamorphic, and sedimentary rocks in the earth's crust. These fluid-saturated microcracks are the most compliant elements of the rock-mass and control rock deformation. A fundamental revision of conventional fluid-rock deformation is identified as New Geophysics (Crampin and Gao, 2008) with implications for almost all solid-earth geosciences, including hydrocarbon exploration and production, and earthquake forecasting. However, there remains a scope to modify Biot's theory with the changes in elastic and dynamical constants for the presence and modification of cracks, beyond the definition of microcracks considered in Hudson's formulation. Moreover, the wave-induced fluid-flow from the surface pores to the adjoining medium may be analysed for the changes in crack parameters. Such an induced flow plays an important role in the diagnosis of an earthquake preparation region.

The work presented studies the problem of Rayleigh waves propagation in a fluid-saturated porous solid pervaded with microcracks. The effects of presence, modification and interconnection of cracks as well as closing of surface pores, are calculated on the existence and phase velocity of Rayleigh waves.

\section{Fundamental Equations}

Following Biot (1956, 1962a,b), a set of differential equations governs the particle motion in an isotropic porous solid frame saturated by a non-viscous fluid. These equations, in the absence of body forces, are given by

$$
\begin{aligned}
& \tau_{i j, j}=\rho \ddot{u}_{i}+\rho_{f} \ddot{w}_{i}, \\
& \left(-p_{f}\right)_{, i}=\rho_{f} \ddot{u}_{i}+m \ddot{w}_{i},
\end{aligned}
$$

where $\tau_{i j}$ and $p_{f}$ are the stress components in porous aggregate and fluid pressure, respectively. The $u_{i}$ are the components of the displacements for the solid and $w_{i}$ are the components of average displacement of fluid relative to the solid. Indices can take the values 1, 2 and 3. Summation convention is valid for repeated indices. The comma (,) before an index represents partial space differentiation and dot over a variable represents partial time derivative. The $\rho$ and $\rho_{f}$ are the densities of porous aggregate and pore-fluid, respectively. The inertial parameter $m$ controls dynamical coupling between fluid and solid phases.

The stresses in the isotropic solid matrix of porous aggregate, following Biot (1962a), are defined as

$$
\sigma_{i j}=\left(\lambda u_{k, k}\right) \delta_{i j}+\mu\left(u_{i, j}+u_{j, i}\right)
$$


and these are related to $\tau_{i j}$ by

$$
\tau_{i j}=\sigma_{i j}+\alpha\left(-p_{f}\right) \delta_{i j},
$$

through the parameter $\alpha$ to represent the elastic coupling between the two constituents. $\delta_{i j}$ is Kronecker delta. Finally, using the above relations, the stresses in the porous aggregate and pore-fluid, are expressed as

$$
\begin{aligned}
& \tau_{i j}=\left[\left(\lambda+\alpha^{2} M\right) u_{k, k}+\alpha M w_{k, k}\right] \delta_{i j}+\mu\left(u_{i, j}+u_{j, i}\right) \\
& -p_{f}=M\left(\alpha u_{k, k}+w_{k, k}\right),
\end{aligned}
$$

where $\lambda, \mu, M$ are the elastic constants. The coefficients $\lambda, \alpha$ and $M$ can be expressed in terms of measurable quantities as follows.

$$
\lambda=K_{s}-\frac{2}{3} \mu, \quad M=\frac{K_{s}}{\gamma K_{s}+\alpha}, \quad \alpha=1-\frac{K}{K_{s}}, \gamma=f\left(\frac{1}{K_{f}}-\frac{1}{K_{s}}\right),
$$

where $f$ denotes porosity and $K_{s}, K_{f}, K$ are the bulk moduli of solid grains, pore-fluid and porous aggregate respectively. Three waves (two dilatational and one shear) propagate in a saturated porous medium. Following Sharma (1996), the velocities of dilatational waves (called $P_{f}, P_{s}$ ) are written as

$$
\alpha_{j}=\frac{\lambda+2 \mu}{\rho_{j}}, \rho_{j}=\frac{B+(-1)^{j} \sqrt{B^{2}-4 A C}}{2 M} ;(j=1,2),
$$

where $A=(\lambda+2 \mu) M, B=\rho M+m\left(\lambda+2 \mu+\alpha^{2} M\right)-2 \rho_{f} \alpha M, C=\rho m-\rho_{f}^{2}$. The $\rho=(1-f) \rho_{s}+f \rho_{f}$ defines the density of porous aggregate in terms of densities of solid grains $\left(\rho_{s}\right)$ and pore-fluid $\left(\rho_{f}\right)$. The velocity of lone $\mathrm{S}$ wave is given by

$$
\beta^{2}=\mu / \rho_{3}, \quad \rho_{3}=\mathrm{C} / \mathrm{m} .
$$

\section{Cracked Poroelastic Solid}

A porous aggregate of bulk modulus $K$ and rigidity modulus $\mu$ is saturated with a fluid of bulk modulus $K_{f}$. It is embedded with fluid-filled circular cracks $(r=r>>d)$, each of radius $r$ and a very small aspect ratio $d / r$. In case these cracks are isolated, the modified elastic constants $(\bar{K}, \bar{\mu})$ of the cracked solid are given by Budiansky and O'Connell (1976),

$$
\frac{\bar{K}}{K}=1-\frac{16}{9} \frac{\left(1-\bar{v}^{2}\right) D \varepsilon}{1-2 \bar{v}}, \frac{\bar{\mu}}{\mu}=1-\frac{32}{45}\left(D+\frac{3}{2-\bar{v}}\right)(1-\bar{v}) \varepsilon,
$$

where, in presence of cracks, the Poisson's ratio $(v)$ of uncracked solid changes to $\bar{v}$. The saturation parameter $D$ and crack density parameter $\varepsilon$ are expressed as follows.

$$
D=\left[1+\frac{4 \Omega}{3 \pi} \frac{K}{\bar{K}} \frac{1-\bar{v}^{2}}{1-2 \bar{v}}\right]^{-1}, \quad \varepsilon=\frac{45}{16} \frac{v-\bar{v}}{1-\bar{v}^{2}} \frac{2-\bar{v}}{D(1+3 v)(2-\bar{v})-2(1-2 v)},
$$

where, for circular cracks, $\Omega=\left(K_{f} / K\right) /(d / r)$ and vanishing of $\Omega$ represents the dry cracks. Following Sharma (1996), the Poisson's ratio $(0<\bar{v}<0.5)$ of the cracked solid is calculated as a real root of the algebraic equation, given by

$$
a_{1} \bar{v}^{5}+a_{2} \bar{v}^{4}+a_{3} \bar{v}^{3}+a_{4} \bar{v}^{2}+a_{5} \bar{v}+a_{6}=0 .
$$

The coefficients $a_{j}$, the functions of $v, \varepsilon$ and $\Omega$, are as follows.

$a_{1}=(1-2 v) \varepsilon\left[2 \varepsilon^{\prime}(1+3 v)\right]+(1+3 v)\left[\frac{9}{8} \varepsilon(1+3 v)-\frac{45}{16} \varepsilon^{\prime}\right], \quad \varepsilon^{\prime}=\varepsilon+\frac{3}{4} \frac{\Omega}{\pi}$, 


$$
\begin{aligned}
& a_{2}=(1-2 v) \varepsilon\left[4 \varepsilon(1-2 v)-\frac{45}{4}+(1+3 v)\left(\frac{9}{4}-4 \varepsilon^{\prime}\right)\right]+\frac{2025}{256}+(1+3 v)\left[\frac{45}{16} \varepsilon^{\prime}(v+4)-\frac{405}{128}-\frac{81}{16} \varepsilon(1+3 v)\right] \\
& a_{3}=(1-2 v) \varepsilon\left[\frac{45}{4}(v+2)+(1+3 v)\left(\frac{-45}{8}-4 \varepsilon^{\prime}\right)\right]-\frac{2025}{128}(v+2)+(1+3 v)\left[-\frac{45}{16} \varepsilon^{\prime}(3+4 v)+\frac{405}{256}(9+2 v)\right. \\
& \left.+\frac{45}{8} \varepsilon(1+3 v)\right] \\
& a_{4}=(1-2 v) \varepsilon\left[(1-2 v)\left(-8 \varepsilon+\frac{45}{4}\right)+8 \varepsilon^{\prime}(1+3 v)\right]+\frac{2025}{256}\left(v^{2}+8 v+4\right)+(1+3 v)\left[-\frac{45}{16} \varepsilon^{\prime}(4-3 v)-\frac{1215}{256}(4+3 v)\right. \\
& \left.+\frac{45}{16} \varepsilon(1+3 v)\right], \\
& a_{5}=(1-2 v) \varepsilon\left[-\frac{45}{4}(2+v)+(1+3 v)\left(\frac{45}{8}+2 \varepsilon^{\prime}\right)\right]-\frac{2025}{64} v(v+2)+(1+3 v)\left[\frac{45}{4} \varepsilon^{\prime}(1+v)+(1+3 v)\left(\frac{405}{64}-\frac{27}{4} \varepsilon\right]\right), \\
& a_{6}=(1-2 v) \varepsilon\left[\frac{45}{2} v+4 \varepsilon(1-2 v)+(1+3 v)\left(-\frac{9}{4}-4 \varepsilon^{\prime}\right)\right]+\frac{2025}{64} v^{2}+(1+3 v)\left[-\frac{45}{4} v\left(\frac{9}{16}+\varepsilon^{\prime}\right)+\frac{9}{4} \varepsilon(1+3 v)\right]
\end{aligned}
$$

For given values of $\varepsilon, v, \Omega$, the equation (10) is solved for a value of $\bar{v} \in(0,0.5)$. Then, the elastic constants for porous solid modified due to the presence of fluid-filled cracks are calculated for the newly calculated Poisson's ratio $\bar{v}$. The relations (5) enable to calculate the corresponding values of $\alpha, \gamma$ and $M$. The presence of cracks in a solid are classified in three categories on the basis of the interconnection between cracks. The following section explains the modifications in the elastic and dynamical properties of the cracked material in these categories.

\subsection{Crack Interconnection Regimes}

Fluid-filled pores are considered to be inherited since the formation of sedimentary rocks. These pores are small and interconnected. However, the cracks appear later with the accumulation of tectonic stresses. Initially, these cracks may be isolated and dry as well. With lapse of time, the fluid-filled connected pores may squeeze out their fluid to fill these cracks. With the further increase of stress, the newly formed cracks may help to connect the isolated cracks. The ultimate dilatancy may force the connected cracks to be drained. Following Budiansky and O'Connell (1976), different interconnection regimes of cracks are considered and corresponding modifications in elastic and dynamical parameters are explained as follows.

a) Saturated Isolated Cracks

The term porosity in Biot's theory means the effective porosity that considers only the connected void space that could be substituted with a liquid. On the contrary any smaller but sealed void space is considered to be the part of the elastic structure. The cracks are introduced and then modified with the accumulation of stress in an elastic material. The stress induced modifications in the shape and size of cracks may end up with the squeezing the boundary pores of a crack. This may prevent the communication of fluid pressure between modified cracks and the host porous solid. Such isolated cracks reduces the interconnected void space in the porous material. In this case the effective porosity (i.e., inter-connected fluid communicating space), reduces with the presence of isolated crack space and is given by

$$
\bar{f}=f-f_{c}, \quad f_{c}=\frac{4 d}{3 r} \pi \varepsilon,
$$

where the $f_{c}$ measures the volume occupied by circular cracks of density $\varepsilon$ and aspect ratio (thickness to radius) $d / r$. The constants and parameters relevant to the elastic characteristics of the medium are modified as follows.

$$
\begin{aligned}
& \bar{K}=\left[1-\frac{16}{9} \frac{\left(1-\bar{v}^{2}\right) D \varepsilon}{1-2 \bar{\nu}}\right] K, \quad \bar{\mu}=\left[1-\frac{32}{45}\left(D+\frac{3}{2-\bar{v}}\right)(1-\bar{v}) \varepsilon\right] \mu, \\
& \bar{M}=\frac{K_{s}}{\bar{\gamma} K_{s}+\bar{\alpha}}, \quad \bar{\alpha}=1-\frac{\bar{K}}{K_{s}}, \quad \bar{\gamma}=\bar{f}\left(\frac{1}{K_{f}}-\frac{1}{K_{s}}\right) .
\end{aligned}
$$

The dissipation parameter $D$ is calculated from the relation

$$
D \varepsilon\left(1-\bar{v}^{2}\right)=\frac{45}{16} \frac{v-\bar{v}}{1+3 v}+2 \varepsilon \frac{\left(1-\bar{v}^{2}\right)(1-2 v)}{(1+3 v)(2-\bar{v})} .
$$


b) Saturated Isobaric Cracks

The fluid-pressure in all the cracks is assumed same throughout the porous medium. This may be possible with the presence of inter-crack communication. In other words, the crack dilatancy intensifies to the extent that all the cracks get connected to each other. The effective Poisson's ratio, for given values of $v$ and $\varepsilon$, is calculated from the relation

$$
\varepsilon=\frac{45}{16} \frac{(2-\bar{v})(v-\bar{v})}{\left(1-\bar{v}^{2}\right)(10 v-\bar{v}-3 v \bar{v})} .
$$

The following relations are used to modify the elastic coefficients.

$$
\bar{K}=K, \quad \bar{\mu}=\left[1-\frac{32}{45} \frac{(1-\bar{v})(5-\bar{v})}{(2-\bar{v})} \varepsilon\right] \mu .
$$

c) Drained Cracks

This may be the ultimate state of the dilatancy of cracks. The cracks are extended up to the outer surface and discharge of saturating fluid is possible from the sample. The elastic material behaves as if no fluid is there, which is equivalent to the one embedded with dry cracks. The modified Poisson's ratio is given by (14) but the modified elastic constants are calculated from relations (12) with $D=1$.

\section{Free Surface of Porous Solid}

Surface of the porous solid is considered a plane identified with its normal $\hat{n}$. A free surface is considered to be free of stress. Hence, at every point on the free surface of porous material the resultant energy must vanish (Deresiewicz and Skalak, 2008). This can be achieved through

$$
\sum_{j=n, t}\left[\tau_{n j} \dot{u}_{j}+s_{n n} \dot{w}_{n}\right]=0
$$

at every point on the surface of material body. The indices $n$ and $t$ identify, respectively, the components of tensors along $\hat{n}$ and in-plane parallel to free surface. Then the boundary conditions, appropriate for a free surface, are given by

$$
\text { i) } \tau_{n n}=0 \text {; ii) } \tau_{n t}=0 \text {; iii) } s_{n n}=Z \dot{w}_{n},
$$

where, $Z$ (a constant) may be termed as a surface flow impedance for the pore-fluid (Denneman, 2002). The value $Z=0$ corresponds to fully open pores (i.e., $s_{n n}=0$ ) and $Z \rightarrow \infty$ corresponds to sealed pores (i.e., $w_{n}=0$ ).

Another parameter $\varepsilon$ may be used to represent the opening of surface pores of the material. Then, $Z$ will be assumed a non-zero, finite constant value for surface flow impedance. The extreme values of $\varepsilon$, i.e., 0 and 1 , denote sealed pores and open pores, respectively. The intermediate values of $\varepsilon$ may represent some restricted or partial opening of pores. The last condition is then written as

$$
\varepsilon S_{n n}=Z(1-\varepsilon) \dot{w}_{n} .
$$

The use of $\varepsilon$, as above, has a special significance. This implies that even a small or restricted opening to the interconnected pores may cause a large drop in the normal stress of pore-fluid. In case of partial opening of pores (i.e., $0<\varepsilon<1$ ), the condition iii) in (17) is replaced with the condition (18). Then we have

$$
\text { i) } \tau_{n n}=0 \text {; ii) } \tau_{n t}=0 \text {; iii) } \varepsilon s_{n n}=Z(1-\varepsilon) \dot{w}_{n},
$$

as the appropriate generalised boundary conditions for the free surface of a saturated porous solid.

\section{Motion in a Plane}

In Cartesian coordinate system $(x, y, z)$, the plane $z=0$ serves as the surface of the porous solid half space occupying the region $Z>0$. The medium being isotropic provides a facility to study the wave motion confined to a plane without losing any information. Hence, the wave motion is studied in $x-Z$ plane and all the quantities are independent of $y$-coordinate. We have $u=\left(u_{x}, 0, u_{z}\right)$ and $w=\left(w_{x}, 0, w_{z}\right)$ and these displacement components are expressed as follows.

$$
\begin{aligned}
& u_{x}=\frac{\partial \phi_{1}}{\partial x}+\frac{\partial \phi_{2}}{\partial x}+\frac{\partial \phi_{3}}{\partial z} ; u_{z}=\frac{\partial \phi_{1}}{\partial z}+\frac{\partial \phi_{2}}{\partial z}-\frac{\partial \phi_{3}}{\partial x} ; \\
& w_{x}=\mu_{1} \frac{\partial \phi_{1}}{\partial x}+\mu_{2} \frac{\partial \phi_{2}}{\partial x}+\mu_{3} \frac{\partial \phi_{3}}{\partial z} ; w_{z}=\mu_{1} \frac{\partial \phi_{1}}{\partial z}+\mu_{2} \frac{\partial \phi_{2}}{\partial z}-\mu_{3} \frac{\partial \phi_{3}}{\partial x} ;
\end{aligned}
$$


where $\mu_{j}=\frac{\rho_{f} \alpha-\rho+\rho_{j}}{\rho_{f}-m \alpha},(j=1,2)$ and $\mu_{3}=\frac{-\rho_{f}}{m}$. The displacement potentials $\phi_{j},(j=1,2,3)$ represent the propagation of $P_{f}, P_{s}$ and $S V$ waves in porous medium, with velocities $\alpha_{1}, \alpha_{2}$ and $\beta$ respectively.

The boundary conditions appropriate for particle motion in $X-Z$ plane are considered at the plane surface $Z=0$. This surface is considered to be free of stress, which implies that normal stress (i.e., $\tau_{z z}$ ) and tangential stress (i.e., $\tau_{z x}$ ) should vanish. One more condition is to be satisfied at this surface, which is derived from the exposure of pore-fluid in the surface pores. The opening of surface pores are ensured through the vanishing of fluid pressure (i.e., $p_{f}$ ) at the plane surface $Z=0$. The sealed surface pores, on the other hand, disallow the seepage of pore-fluid out of the solid matrix.

\section{Rayleigh Waves}

For the propagation of harmonic plane waves along the x-direction decaying exponentially in z-direction, the displacement potentials are chosen as follows.

$$
\phi_{j}=A_{j} e^{i k(x-c t)-k d_{j} z},(j=1,2,3),
$$

where $k$ is horizontal wave number, $c$ is apparent phase velocity and $d_{j}=\sqrt{1-c^{2} / \alpha_{j}^{2}},(j=1,2), d_{3}=\sqrt{1-c^{2} / \beta^{2}}$.

Using these potentials, the displacements of solid and fluid particles are calculated from relations (20). Then relations (4) are employed to calculate the stresses in the porous medium. Thus the calculated displacements and stresses are subjected to boundary conditions as follows.

a) Fully-opened surface pores

The appropriate boundary conditions to be satisfied at the stress-free surface $Z=0$, in this case, are given by

$$
\text { i) } \tau_{z z}=0 \text {, ii) } \tau_{z x}=0 \text {, iii) } p_{f}=0 .
$$

The condition for the existence of Rayleigh waves in saturated porous medium is specified through a secular equation, given by

$$
4 \sqrt{1-\frac{c^{2}}{\beta^{2}}}\left(\sqrt{1-\frac{c^{2}}{\alpha_{1}^{2}}}-\eta \sqrt{1-\frac{c^{2}}{\alpha_{2}^{2}}}\right)=\left(2-\frac{c^{2}}{\beta^{2}}\right)\left[\left(2-\frac{\rho_{1}}{\rho_{3}} \frac{c^{2}}{\beta^{2}}\right)-\eta\left(2-\frac{\rho_{2}}{\rho_{3}} \frac{c^{2}}{\beta^{2}}\right)\right],
$$

where $\eta=\frac{\alpha+\mu_{1}}{\alpha+\mu_{2}}\left(\frac{\alpha_{2}}{\alpha_{1}}\right)^{2}$.

With the substitution of $\eta=0$ and $\rho_{3}=\rho_{1}=\rho$, the equation (23) reduces to the secular equation for propagation of classical Rayleigh waves in perfectly elastic solid.

b) Sealed surface pores

In this case, the boundary conditions includes the restriction on the drainage of pore-fluid at the surface $Z=0$. hence the appropriate boundary conditions to be satisfied are

$$
\text { i) } \tau_{z z}=0 \text {, ii) } \tau_{z x}=0 \text {, iii) } \dot{w}_{z}=0 \text {. }
$$

With these boundary conditions the secular equation obtained for existence of Rayleigh waves in porous solid half space is given by

$$
\begin{aligned}
& 4 \sqrt{1-\frac{c^{2}}{\alpha_{1}^{2}}}\left(1-\eta_{1}\right) \sqrt{1-\frac{c^{2}}{\beta^{2}}}+\eta_{2}\left(\sqrt{1-\frac{c^{2}}{\alpha_{1}^{2}}}-\sqrt{1-\frac{c^{2}}{\alpha_{2}^{2}}}\right)= \\
& \left(2-\frac{c^{2}}{\beta^{2}}\right)\left[\left(2-\frac{\rho_{1}}{\rho_{3}} \frac{c^{2}}{\beta^{2}}\right)-\eta_{1}\left(2-\frac{\rho_{2}^{\prime}}{\rho_{3}} \frac{c^{2}}{\beta^{2}}\right) \frac{\sqrt{1-\frac{c^{2}}{\alpha_{2}^{2}}}}{\sqrt{1-\frac{c^{2}}{\alpha_{2}^{2}}}}+2 \frac{\eta_{2}}{\rho_{3}}\left(\frac{\lambda_{1}}{\alpha_{1}^{2}}-\frac{\lambda_{2}}{\alpha_{2}^{2}} \sqrt{\left.\frac{1-\frac{c^{2}}{\alpha_{1}^{2}}}{1-\frac{c^{2}}{\alpha_{2}^{2}}}\right) \frac{c^{2}}{\beta^{2}}},\right.\right.
\end{aligned}
$$


where $\eta_{1}=\frac{\mu_{1}}{\mu_{2}}, \eta_{2}=\frac{\mu_{3}}{\mu_{2}}$ and $\lambda_{j}=\lambda+\alpha M\left(\alpha+\mu_{j}\right),(j=1,2)$.

With the substitution of $\eta_{1}=\eta_{2}=0$ and $\rho_{1}^{\prime}=\rho_{3}=\rho$, the equation (25) reduces to the secular equation for propagation of classical Rayleigh waves in perfectly elastic solid.

\section{Numerical Example}

The main objective of this study is to observe the effect of the presence, modifications and saturation of cracks on the velocity of Rayleigh waves. But, the relations for elastic characteristics in the previous sections are not very simple to be explored analytically. Moreover, the secular equations derived for propagation of Rayleigh waves can not be solved through algebraic methods. Hence, we consider a numerical example to understand the role of cracks and pores on the propagation of Rayleigh waves. Following the experimental results of Fatt (1959) for kerosene-saturated sandstone, we choose the following values for the relevant parameters: $K=4.6 G P a, \mu=2.7 G P a, K_{s}=6 G P a, K_{f}=2.14 G P a$,

$$
\rho_{s}=2100 \mathrm{~kg} / \mathrm{m}^{3}, \quad \rho_{f}=1000 \mathrm{~kg} / \mathrm{m}^{3}, f=0.26, m=1.1 \rho_{f} / f \text {. }
$$

The porous sandstone is assumed to be embedded with circular cracks identified with the fixed values of aspect ratio $(d / r)$ and density $(\varepsilon)$.

Using the above parameters, the velocities $\left(\alpha_{1}, \alpha_{2}, \beta\right)$ of three body waves and phase velocity of Rayleigh waves (i.e., $\left.C\right)$ are computed for fully open as well as sealed surface pores of the porous solid. The variations of velocities with crack density $(\varepsilon)$ are shown in Figure 1 and the corresponding variations aspect ratio $(d / r)$ are shown in Figure 2. Other two figures display the effect of modifications of cracks through three interconnected crack regimes. Here follows the detailed discussion of the plots in these figures.
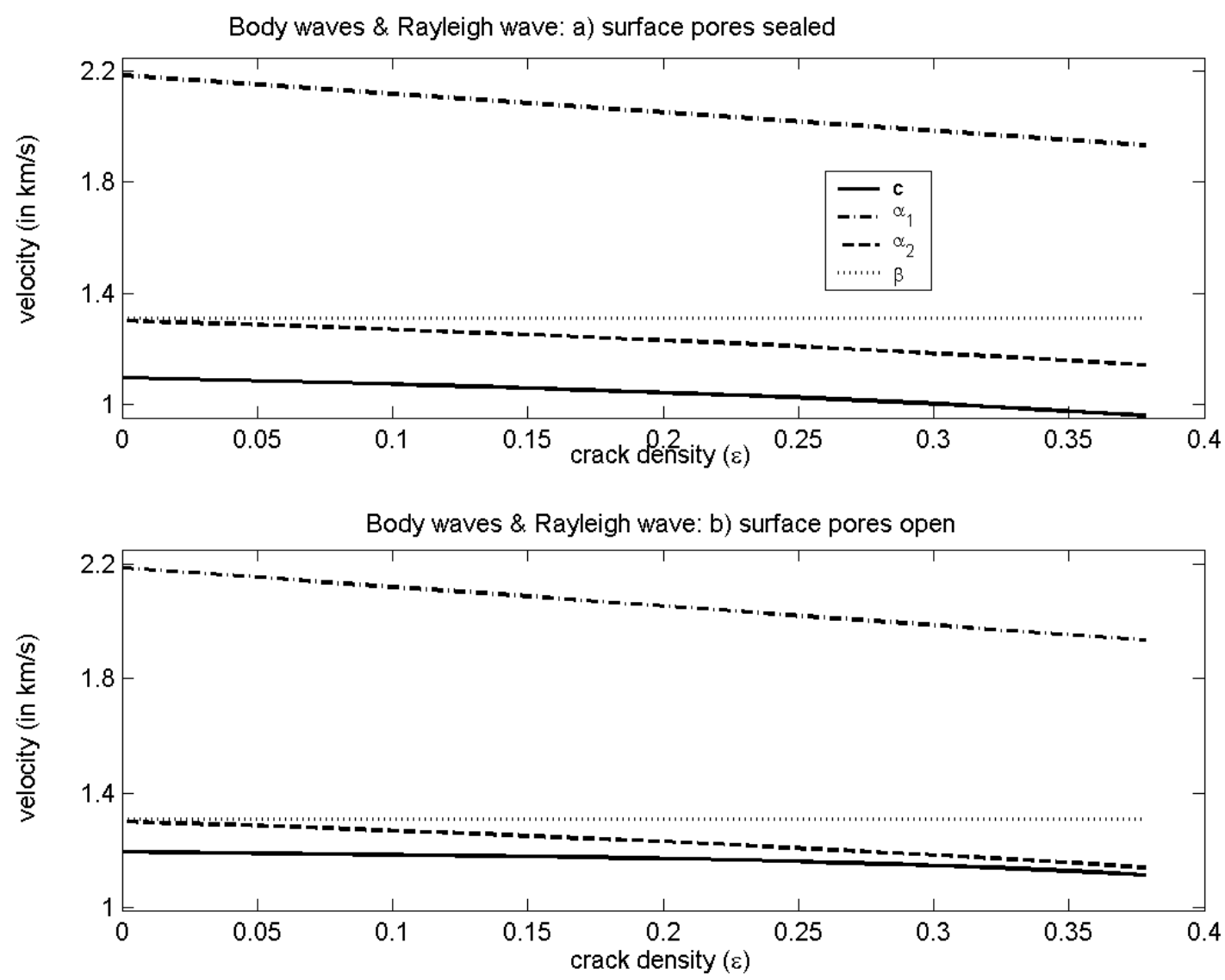

Figure 1: Variations of velocities of body waves and Rayleigh wave with crack density; $(d / r=0.001)$. 
Figure 1: Plots in this figure exhibit the variations of velocities of body $\left(P_{f}, P_{s}, S\right)$ as well surface (Rayleigh) waves with the crack density. The crack density is varied with $\varepsilon \in(0,0.38)$. The aspect ratio of circular shaped cracks is fixed with thicknessto-radius ratio as $d / r=0.001$. The surface pores are either sealed or fully opened. It is noted that irrespective of pore-opening status, the velocities of $P_{f}, P_{s}$ and Rayleigh waves decrease with the increase of crack density. However, this effect is negligible on the velocity $(\beta)$ of $\mathrm{S}$ waves. It is further noted that the velocities $\left(\alpha_{1}, \alpha_{2}, \beta\right)$ of body waves are not affected with the opening or closing of the surface pores. But, the velocity $(C)$ of Rayleigh waves is significantly larger when the surface pores are open. While comparing the Rayleigh velocity curves in two parts of this figure, it may be noted that decrease of $C$ with $\varepsilon$ is more steady when surface pores are open.

Figure 2: The variations of velocities $\left(\alpha_{1}, \alpha_{2}, \beta\right)$ of body waves and velocity ( $C$ ) of Rayleigh waves with the thickness of circular cracks are shown in this figure. The value of crack density is fixed as $\varepsilon=0.2$ and aspect ratio is varied as $d / r \in(0,0.01)$. Surface pores keep the option of either sealed or fully opened. It is noted that whether the surface pores are opened or sealed, the extent of crack thickness have negligible effect on the velocities of body $\left(P_{f}, P_{s}, S\right)$ waves or the surface (Rayleigh) waves. Comparison of the solid-line curves in two parts of this figure shows that faster propagation of Rayleigh waves due to the opening of surface pores remains unaffected with any change in the aspect ratio of circular cracks.
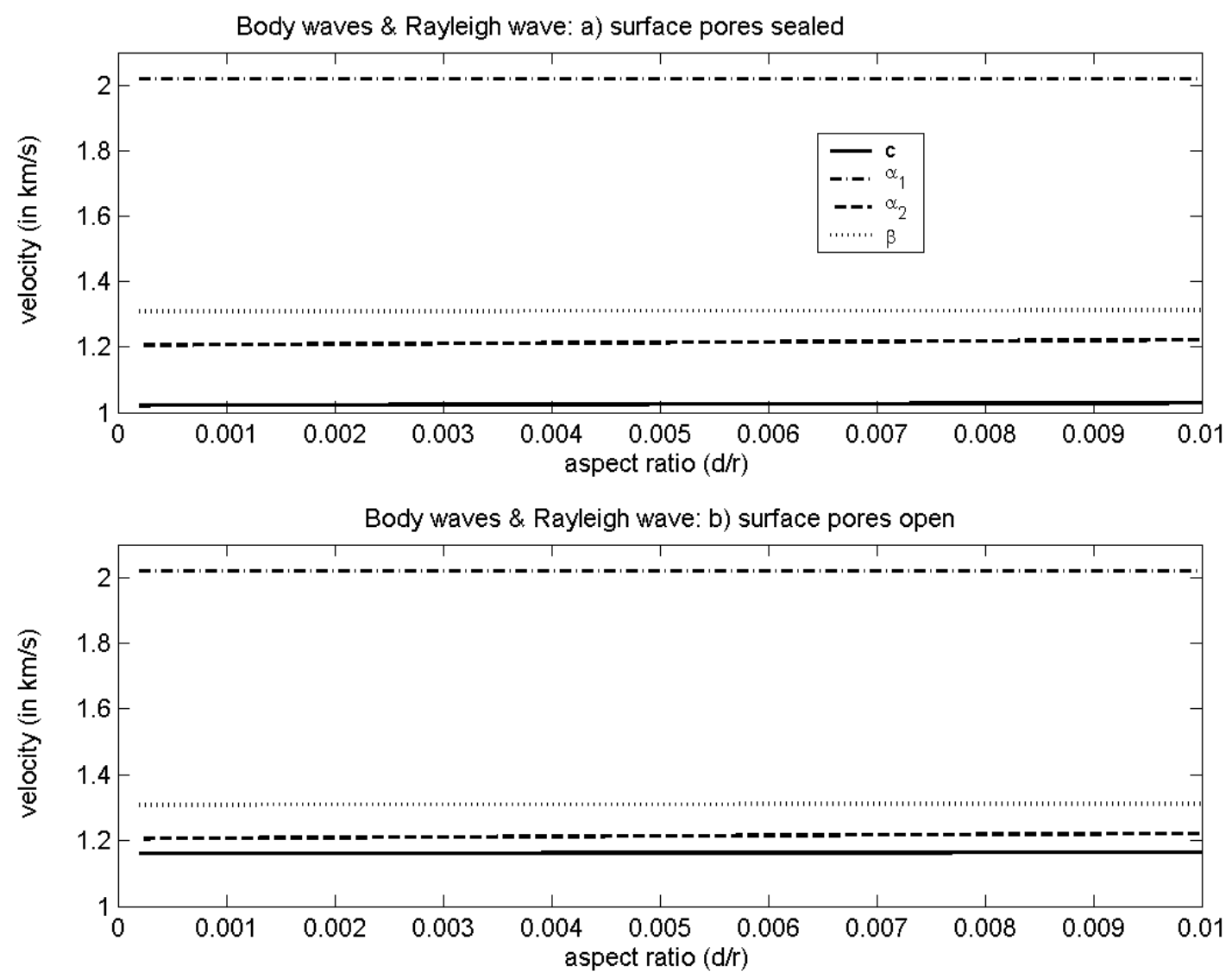

Figure 2: Same as the Figure 1 but variations with aspect ratio; $(\varepsilon=0.2)$.

Figure 3: In general, the phase velocity of classical Rayleigh waves is analysed through its ratio $(c / \beta)$ with the velocity of S (or shear) wave. But in porous medium, the $P_{s}$ wave is, generally, found to be the slowest wave. This implies that a valid Rayleigh waves in porous medium should propagate with velocity lower that the velocity $\left(\alpha_{2}\right)$ of the slowest body wave. Keeping 
this in mind, the non-dimensional phase velocity (i.e. ratio $\left.c / \alpha_{2}\right)$ is plotted in figure 3. Its variations with $\varepsilon \in(0,0.38)$ are plotted for three interconnection crack regimes (i.e, Isolated: saturated isolated cracks; Isobaric: saturated isobaric cracks; Drained: drained cracks). The aspect ratio of circular shaped cracks is fixed as $d / r=0.001$. The surface pores are either sealed or are fully-opened. From the plots in this figure, it is noted that a change in crack density may affect the propagation of Rayleigh waves most when cracks are interconnected but drained. This implies that the presence of fluid may mitigate the effect of cracks on the (non-dimensional) velocity of surface waves. In case of sealed surface pores, the effect of crack density on $c / \alpha_{2}$ is least when the embedded cracks are saturated but isolated. On the other hand, in case of fully-opened surface pores, the effect of crack density on $c / \alpha_{2}$ is least when the embedded cracks are interconnected and saturated, i.e. isobaric.
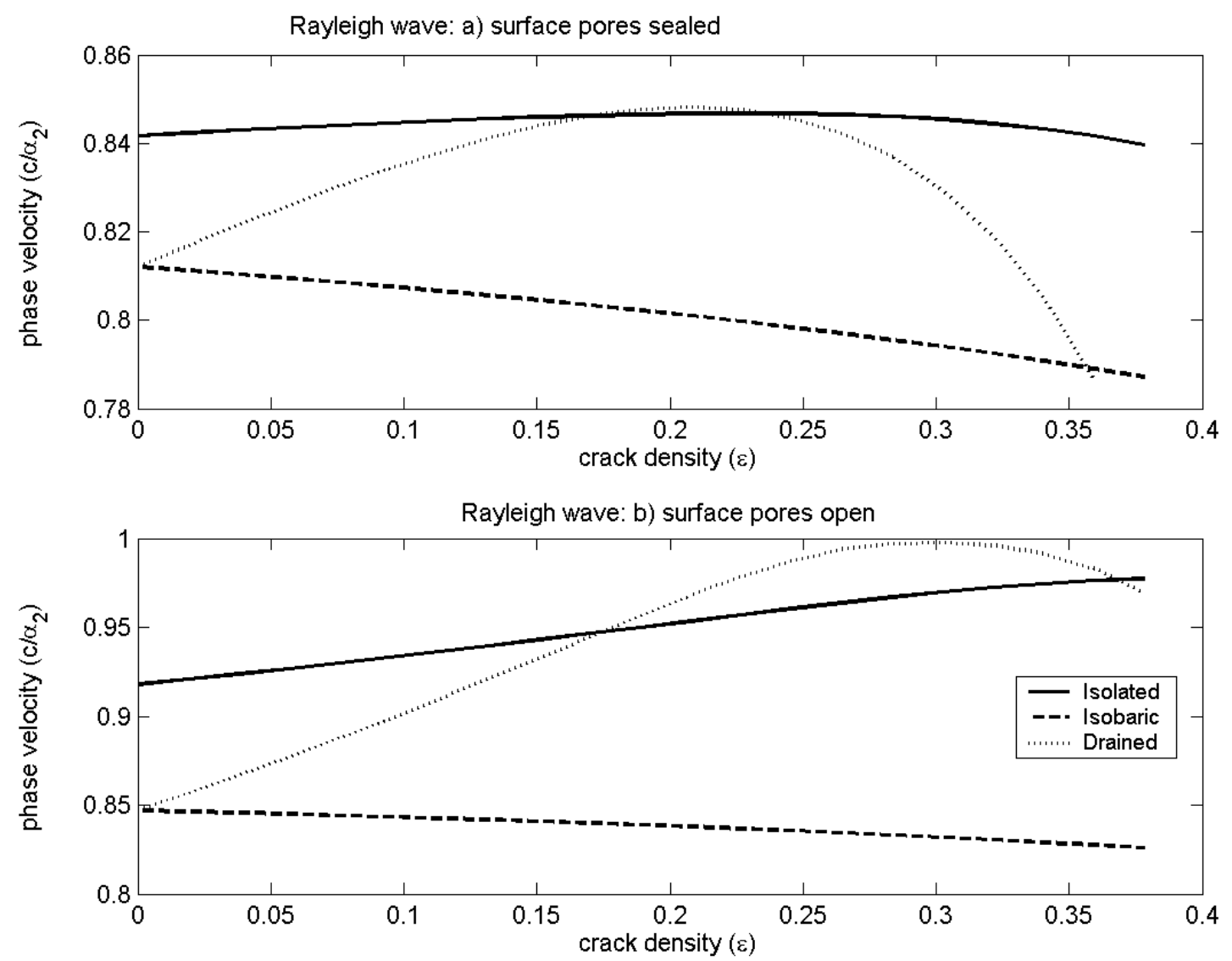

Figure 3: Variations of Rayleigh wave velocity with crack density;

$(d / r=0.001)$; crack regimes: saturated Isolated cracks, saturated Isobaric cracks, Drained cracks.

Amongst three crack regimes considered, the presence of saturated isobaric cracks indicates the smallest value of ratio $c / \alpha_{2}$, whether the surface pores are opened or not. However, in general, the larger values of this ratio may be expected for isolated cracks when surface pores are sealed and for drained cracks when surface pores are open. Overall, in any of the three crack regimes, this velocity ratio $\left(c / \alpha_{2}\right)$ is larger when the surface pores are open.

Figure 4: Modifications of cracks leading to different crack regimes may be caused mainly with the changes in aspect ratio $d / r$ (thickness-to-radius ratio) of embedded cracks. Hence, in the figure 4, the variations of non-dimensional phase velocity (i.e. ratio $\left.c / \alpha_{2}\right)$ are displayed with $d / r \in(0,0.01]$. Note that, whatever is the aspect ratio and whichever is the crack regime, the velocity ratio $\left(c / \alpha_{2}\right)$ is larger when the surface pores are open. Changes in aspect ratio may not affect the values of the velocity ratio to a noticeable extent, particularly when cracks are saturated isobaric. However, velocity of surface waves may affect a little with a change of $d / r$, when the cracks are isolated or drained. 

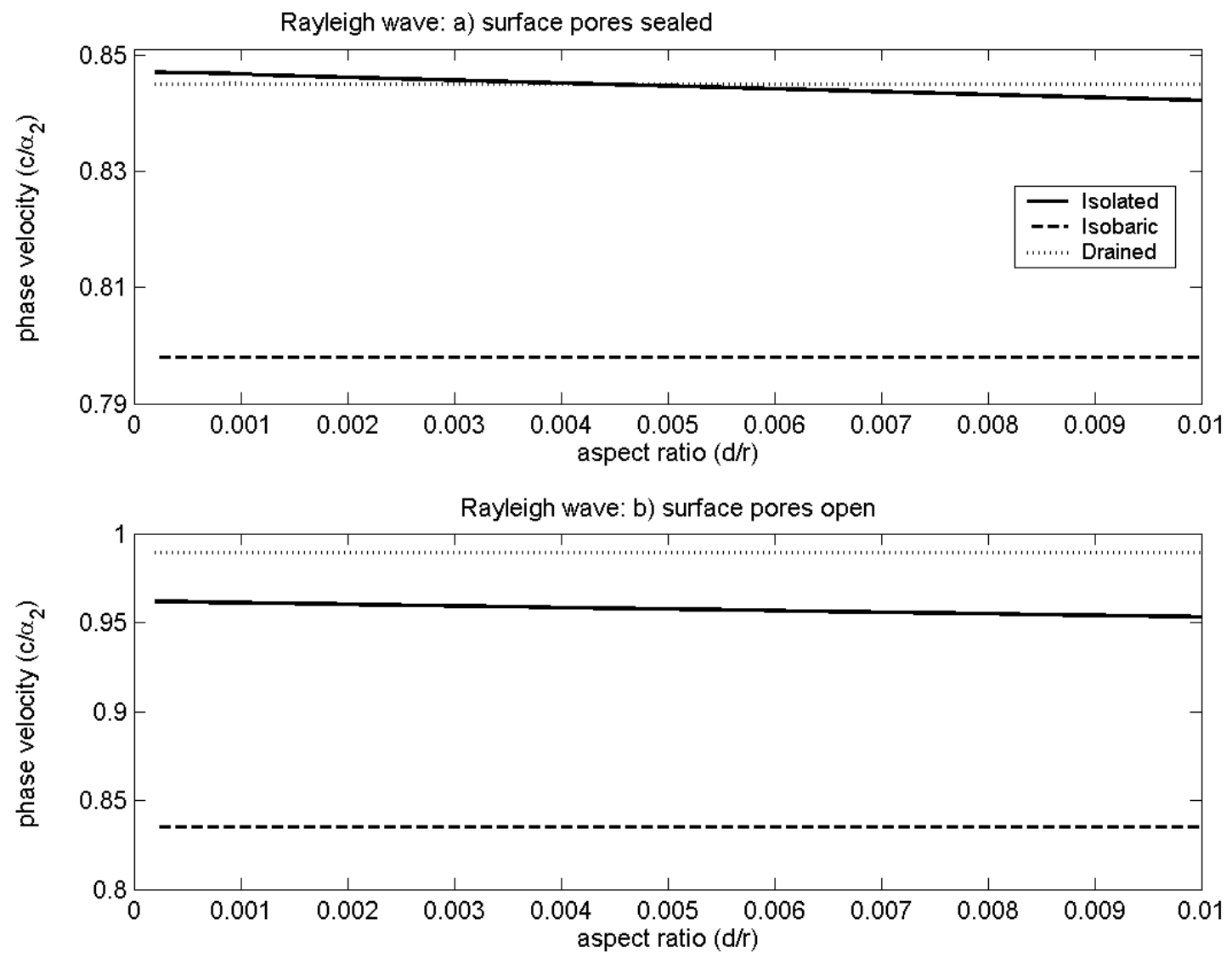

Figure 4: Same as the Figure 3 but variations with aspect ratio; $(\varepsilon=0.2)$

The work presented considers the propagation of seismically significant Rayleigh waves in a realistic model of the crust. This improves upon a much earlier work of Tajuddin (1984) with the presence of dilatant cracks and using the more realistic boundary conditions. The numerical example explains the importance of crack characteristics (i.e. density, thickness-to-radius ratio and connections among cracks) on the velocity of Rayleigh waves as well as body waves in the cracked poroelastic medium.

\section{References}

Biot M. A., 1956. The theory of propagation of elastic waves in a fluid-saturated porous solid, I. Low-frequency range, II. Higher frequency range. J. Acoust. Soc. Am., Vol. 28, pp. 168-191.

Biot M. A., 1962a. Mechanics of deformation and acoustic propagation in porous media. J. Appl. Phys., Vol. 33, pp. 1482-1498.

Biot M. A., 1962b. Generalized theory of acoustic propagation in porous dissipative media. J. Acoust. Soc. Am., Vol. 34, pp/ 1254-1264.

Budiansky B., and R J. O'Connell, 1976. Elastic moduli of dry and saturated cracked solids. Int. J. Solids Struct., Vol. 12, pp. 8197.

Crampin S., 1978. Seismic wave propagation through a cracked solid : polarisation as a possible dilatancy diagnostic. Geophys. $J$. R. Astr. Soc., Vol. 53, pp. 467-496.

Crampin S., 1984. Effective elastic-constants for wave propagation through elastic solids. Geophys. J. R. Astr. Soc., Vol. 76, pp. 135-145.

Crampin S., 1985. Evidence for aligned cracks in Earth's crust. First Break, Vol. 3, pp. 12-15.

Crampin S., 1987. The basis for earthquake prediction. Geophys. J. R. Astr. Soc., Vol. 91, pp. 331-347.

Crampin S., 2006. The new geophysics: A new understanding of fluid-rock deformation. EUROCK 2006 - Multiphysics Coupling and Long Term Behaviour in Rock Mechanics, (Edited by Van Cotthem A., Charlier R., Thimus J.F. and Tshibangu J.P.), Taylor and Francis, London, pp. 539-544.

Crampin S., and Y. Gao, 2008. A review of the new geophysics: A new understanding of pre-fracturing deformation in the crackcritical crust with implications for hydrocarbon production. Pet. Sci., Vol. 5, pp. 1-12. 
Deresiewicz R., and R. Skalak, 2008. On uniqueness in dynamic poroelasticity. Bull. Seism. Soc. Am., Vol. 53, pp. 783-789.

Denneman A. I. M., Drijkoningen G.G., D. M. J. Smeulders, and K. Wapenar, 2002. Reflection and transmission of waves at a fluid/porous medium interface. Geophysics, Vol. 67, pp. 282-291.

Eshelby J.D., 1957. The determination of the elastic field of an ellipsoidal inclusions and related problems. Proc. Roy. Soc.-A, 241, pp. 376-396.

Fatt I., 1959. Biot-Willis elastic coefficients for a sandstone. J. Appl. Mech., Vol. 26, pp. 296-297.

Garvin H.D., and Knopoff L., 1973. The compressional modulus of a material permeated by a random distribution of circular cracks. Quart. Appl. Math., Vol. 30, pp. 453-464.

Garvin H.D., and Knopoff L., 1975a. The shear modulus of a material permeated by a random distribution of free circular cracks. Quart. Appl. Math., Vol. 30, pp. 296-300.

Garvin H.D., and Knopoff L., 1975b. Elastic moduli of a medium with liquid filled cracks. Quart. Appl. Math., Vol. 30, pp. 301303.

Hudson J. A., 1980. Overall properties of a cracked solid. Math. Proc. Camb. Phil. Soc., Vol. 88, pp. 371-384.

Hudson J. A., 1981. Wave speed and attenuation of elastic waves in materials containing cracks. Geophys. J. R. Astr. Soc., Vol. 64, pp. 133-150.

Hudson J.A., E. Liu, and S. Crampin, 1996. The mechanical properties of materials with interconnected cracks and pores. Geophys. J. Int., Vol. 124, pp. 105-112.

O’Connell R.J., and Budiansky B., 1974. Seismic velocities in dry and saturated cracked solids. J. Geophys. Res., Vol. 79, pp. 5412-5426.

Sharma M.D., 1996. Surface wave propagation in a cracked poroelastic half-space lying under uniform layer of fluid. Geophys. J. Int., Vol. 127, pp. 31-39.

Sharma M.D., 1999. Reflection and refraction at an interface between cracked elastic solid and ordinary elastic solid. Ind. J. Pure Appl. Math., Vol. 30, pp. 465-476.

Sharma M.D., and T. N. Saini, 2001. Velocity anomalies in a cracked poroelastic region. Ind. J. Pure Appl. Math., Vol. 32, pp. 331-341.

Sharma M.D., 2004a. Three dimensional wave propagation in a general anisotropic poroelastic medium - velocity, group velocity and polarization. Geophys. J. Int., Vol. 156, pp. 329-344.

Sharma M.D., 2004b. Surface waves in a general anisotropic poroelastic medium. Geophys. J. Int., Vol. 159, pp. 703-710.

Singh S.J., and Rani S., 2006. Plain strain deformation of a multilayered poroelastic half space by surface loads. J. Earth Sys. Sci., Vol. 115, pp. 685-694.

Singh S.J., Rani S., and Kumar R., 2007. Quasi-static deformation of a poroelastic half-space with anisotropic permeability by two-dimensional surface loads. Geophys. J. Int., Vol. 170, pp. 1311-1327.

Tajuddin M., 1984. Rayleigh waves in a poroelastic half-space. J. Acoust. Soc. Am., Vol. 75, pp. 682-684.

Vashisth A.K., and Khurana P., 2005. Wave propagation along a cylindrical bore hole in an anisotropic poroelastic solid. Geophys. J. Int., Vol. 161, pp. 295-302.

\section{Biographical notes}

Dr. J. S. Nandal is a Professor in M. D. University, Rohtak, India. He has more than 25 years of experience in post-graduate teaching and research. His current area of research includes elastic wave propagation based on linear differential equations. He has published more than fifty papers in various international journals of repute.

Mr. T. N. Saini is an Associate Professor of Mathematics in P.G. Government College Kalka, India. He has more than 28 years of experience in graduate teaching. $\mathrm{He}$ is also pursuing his $\mathrm{PhD}$ from M. D.University, Rohtak, India.

Received January 2013

Accepted February 2013

Final acceptance in revised form November 2013 